\title{
Thyroid Storm with Acute Flaccid Quadriparesis due to Thyrotoxic Myopathy: A Case Report
}

\author{
Hwee Ching Tee, Serena Sert Kim Khoo, Yin Khet Fung \\ Department of Endocrinology, Queen Elizabeth Hospital II, Kota Kinabalu, Sabah, Malaysia
}

\begin{abstract}
Thyrotoxicosis is a well-recognized cause of myopathy, but rarely presents as acute flaccid quadriparesis. We report a 25-year-old female with underlying uncontrolled Graves' disease who presented with thyroid storm and acute flaccid quadriparesis due to thyrotoxic myopathy. She showed marked clinical improvement with subsequent normalization of her thyroid parameters. Besides highlighting this rare association, this report underscores the importance of considering thyrotoxic myopathy in the evaluation of patients with acute flaccid quadriparesis.
\end{abstract}

Key words: Thyroid storm, quadriplegia, thyrotoxic myopathy, Graves' disease

\section{INTRODUCTION}

Thyroid storm is a potentially life-threatening condition characterized by multi-organ failure due to severe thyrotoxicosis. Neuromuscular weakness often occurs in thyrotoxic state presenting as chronic myopathy. An acute form of myopathy on the other hand is much rarer, manifesting as acute flaccid quadriparesis with respiratory failure. ${ }^{1}$ We report a case of thyroid storm with acute flaccid quadriparesis due to thyrotoxic myopathy, highlighting the importance of rapid diagnosis to prevent further morbidity.

\section{CASE}

A 25-year-old female presented with fever, breathlessness, palpitation and rapidly progressive generalised body weakness rendering her bedridden over a period of 3 days. She has a background of Graves' disease, diagnosed 6 months earlier when she presented with weight loss, palpitation and heat intolerance. Initial investigation revealed severe thyrotoxicosis with thyroid stimulating hormone (TSH) level of $<0.008 \mathrm{mIU} / \mathrm{L}$ (normal range: 0.35-4.94), free thyroxine (FT4) level of $>75 \mathrm{pmol} / \mathrm{L}$ (9.0119.05) and TSH receptor antibody (TRAb) level of 19.3 IU/L $(<1.0)$. She was started on oral carbimazole but her thyrotoxicosis was suboptimally controlled due to poor compliance. She was otherwise well and did not have any previous muscle weakness.

Upon assessment, she appeared agitated with a blood pressure of $102 / 50 \mathrm{mmHg}$ and respiratory rate of $32 / \mathrm{min}$. She was also febrile at $38^{\circ} \mathrm{C}$ and had an irregular pulse rate of 150 beats $/ \mathrm{min}$. Clinical examination revealed a diffuse grade 3 goiter with thyroid bruit and bilateral exophthalmos with inactive thyroid eye disease. She also

ISSN 0857-1074 (Print) I eISSN 2308-118x (Online)

Printed in the Philippines

Copyright (C) 2020 by Tee et al.

Received: December 13, 2019. Accepted: February 14, 2020.

Published online first: April 20, 2020.

https://doi.org/10.15605/jafes.035.01.20 had generalised hypotonia, areflexia and diminished power of Medical Research Council (MRC) grade 2/5 in her upper limbs and $1 / 5$ in her lower limbs both proximally and distally. Her cranial nerve examination and pinprick sensation were normal. Her condition was further complicated by decompensated cardiac failure with poor peripheral perfusion, raised jugular venous pressure, bibasal lung crepitations and gallop rhythm. Electrocardiography showed atrial fibrillation and chest $X$-ray revealed signs of pulmonary congestion and cardiomegaly.

Her thyroid function test (TFT) demonstrated overt hyperthyroidism with TSH level of $<0.01 \mathrm{mIU} / \mathrm{L}$ and FT4 level of $43.56 \mathrm{pmol} / \mathrm{L}$. Her serum potassium level was normal, $4 \mathrm{mmol} / \mathrm{L}$ (3.5- 5.1). Her serum bilirubin level was elevated at $53.5 \mathrm{umol} / \mathrm{L}(3.4-17.1)$ and she had severe transaminitis with alanine aminotransferase of $748 \mathrm{U} / \mathrm{L}(0-55)$ and aspartate aminotransferase of 1755 $\mathrm{U} / \mathrm{L}$ (5-34). t Subsequent investigations including blood culture, lumbar puncture and antiganglioside antibodies were normal. Her nerve conduction study (NCS) showed reduced compound motor action potential (CMAP) amplitudes with normal conduction velocities and distal motor latencies (Figure 1). Her sensory nerve action potentials (SNAP) were within normal limits and this relative sparing of SNAP was suggestive of underlying myopathy. Her electromyography (EMG) showed small amplitudes, short duration motor unit action potential with early recruitment. There were also increased insertional activities with complex repetitive discharges, in keeping with recent onset generalized myopathy (Table 2). Repetitive nerve stimulation was not performed in view of the positive EMG findings.

Her Burch-Wartofsky score was 85, highly suggestive of thyroid storm (Table 1). A diagnosis of thyroid storm 

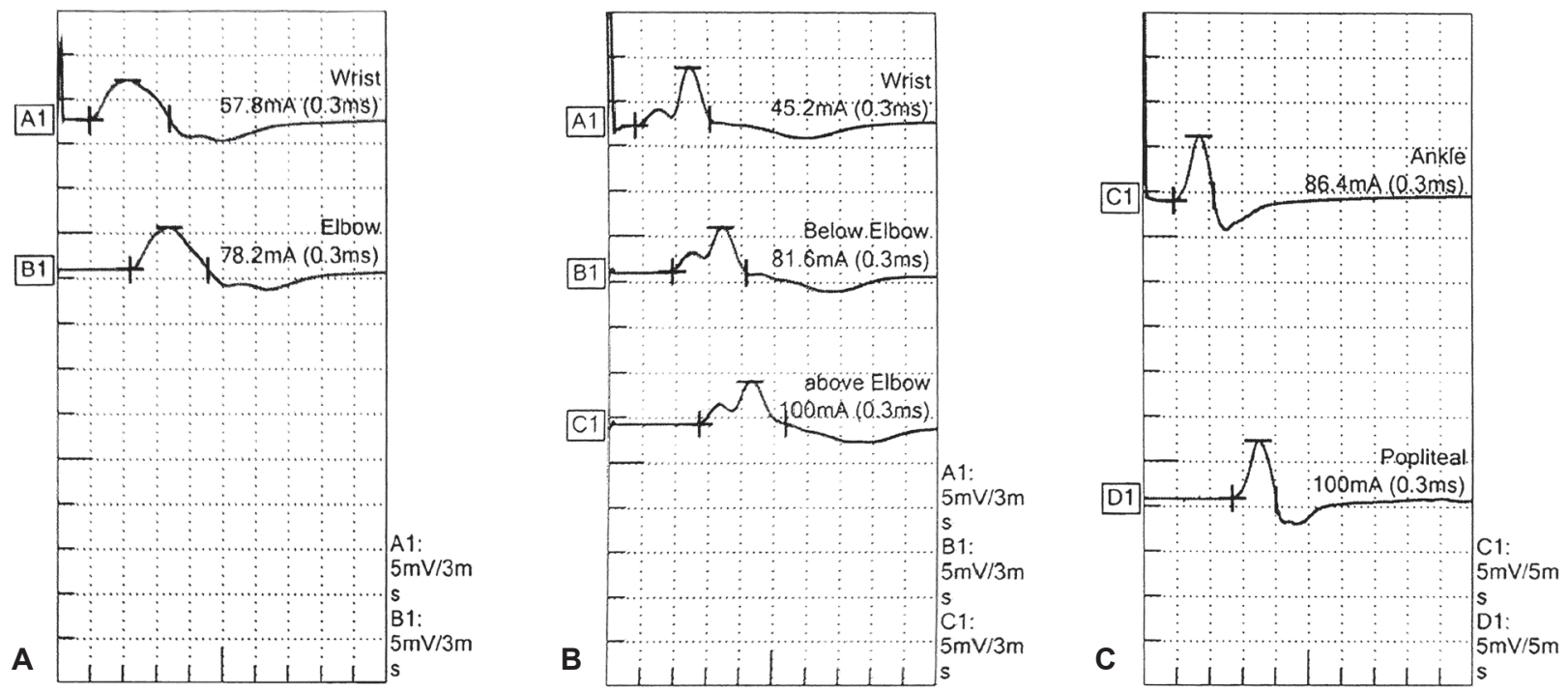

Figure 1. Motor NCS of the patient showed reduced compound motor action potential (CMAP) amplitudes of (A) median, (B) ulnar and (C) tibial nerve.

Table 1. Burch-Wartofsky score of the patient

\begin{tabular}{llc}
\hline & Diagnostic Parameters & Scoring points \\
\hline Thermoregulatory dysfunction & Temperature $38^{\circ} \mathrm{C}$ & 10 \\
Central nervous system effects & Agitation & 10 \\
Gastrointestinal-hepatic dysfunction & Jaundice & 20 \\
Cardiovascular dysfunction: & & \\
$\quad$ Tachycardia & Heart rate: $\geq 140$ beats per minute & 25 \\
$\quad$ Congestive heart failure & Moderate & 10 \\
$\quad$ Atrial fibrillation & Present & 10 \\
Precipitating events & Absent & 0 \\
\hline Total score & & $85^{*}$ \\
\hline
\end{tabular}

*Total score of more than 45 suggestive of thyroid storm

Table 2. EMG of the patient

\begin{tabular}{lccccc}
\hline Muscle group & Insertional Activity & Complex Repetitive Discharges & Amplitude & Duration & Recruitment \\
\hline Deltoid & Normal & - & 0 & Short & Early \\
Biceps brachii & Normal & - & 0 & Short \\
Illiopsoas & Normal & - & 0 & Short \\
Vastus lateralis & Normal & - & -1 & Early \\
Tibialis anterior & Increased & +2 & 0 & Short \\
Gastrocnemius & Increased & +2 & 0 & Short & Short \\
\hline
\end{tabular}

complicated with decompensated cardiac failure, severe hepatitis and acute thyrotoxic myopathy was made. She was intubated for type 1 respiratory failure and was started on intravenous hydrocortisone $100 \mathrm{mg}$ three times daily, oral Lugol's iodine 8 drops three times daily, oral cholestyramine $4 \mathrm{~g}$ four times daily and oral propranolol $40 \mathrm{mg}$ four times daily. Oral carbimazole $20 \mathrm{mg}$ twice daily was subsequently started after 3 days when the liver function tests (LFT) improved. Within 3 days, her condition improved tremendously and she was extubated. Following one week of treatment, her muscle power improved partially to MRC grade $3 / 5$ coinciding with an improvement in her TFT with FT4 of $15.32 \mathrm{pmol} / \mathrm{L}$ and $\mathrm{TSH}<0.01 \mathrm{mIU} / \mathrm{L}$. Total thyroidectomy was performed during the same admission and she was then started on thyroxine replacement. She required assisted ambulation with walking frame initially upon discharge but gradually regained full muscle power over three months following biochemical remission.

\section{DISCUSSION}

Neurological complications of thyrotoxicosis can manifest in the brain all the way down to the muscle, causing encephalopathy, seizures, neuropathy and neuromuscular disorders. Varying degrees of muscular weakness with different aetiologies were reported in up to $82 \%$ of patients with hyperthyroidism. ${ }^{1}$ Acute thyrotoxic myopathy has been long described but is rarely reported, presumably due to the low incidence. This condition usually presents with severe proximal and distal weakness, and rarely quadriplegia with bulbar and respiratory muscle involvement. ${ }^{2}$ Due to the rarity of the condition, the acute weakness is thought to be due to concomitant myasthenia gravis instead of pure myopathy. ${ }^{3}$ The presence of reports in whom myasthenia gravis was excluded however, suggests otherwise. ${ }^{2,4}$

Before reaching a diagnosis of acute myopathy, it is crucial to exclude other causes of lower motor neuron weakness 
in thyrotoxic patients as the treatment differs significantly. Myasthenia gravis (MG) should be the first consideration, especially in patients with bulbar symptoms. The rate of MG was found to be higher in Graves' disease $(0.18 \%)$ than in the general population $(0.01 \%) .{ }^{5}$ However, MG was unlikely in this patient as there were no ocular signs, clinical fatiguability and her EMG was consistent with myopathic changes. Her spontaneous improvement without definitive treatment for MG also was against this diagnosis.

Another differential to consider, Guillain-Barré Syndrome, was also unlikely with the absence of cranial nerve involvement, normal cerebrospinal fluid protein, negative antiganglioside antibody and non-suggestive NCS findings. Hypokalemic periodic paralysis and rhabdomyolysis are widely reported causes of acute paralysis in thyrotoxic patients. Our patient had normal potassium and creatine kinase levels, making these diagnoses improbable. In an intensive care setting, the metabolic derangement associated with hyperthyroidism and intravenous glucocorticoids can increase the risk of critical illness myopathy (CIM) or critical illness polyneuropathy (CIP). ${ }^{6}$ The onset of our patient's weakness which preceded admission and hydrocortisone commencement excludes these 2 conditions.

We arrived at the diagnosis of acute thyrotoxic myopathy in this patient in view of the EMG findings and the marked clinical improvement following normalization of thyroid function. In patients with thyrotoxic myopathy, serum creatine kinase and myoglobin concentrations are usually normal despite muscle wasting and this does not correlate well with the degree of muscular weakness. ${ }^{1}$ The EMG in these patients demonstrate myopathic findings of increased polyphasic, low-amplitude motor unit action potentials and there is usually no spontaneous electrical activities. ${ }^{1}$ Muscle biopsy is rarely required, but if done would reveal various findings, which include normal, non-specific structural alteration and type 2 fiber predominance. ${ }^{1}$

The pathophysiology of muscle disease in thyrotoxicosis is probably multifactorial. Contributing mechanisms may include increased cellular metabolism and energy utilization, increased catabolism and protein degradation, and inefficient energy utilization. ${ }^{7}$ The likelihood of developing weakness is correlated with the duration of the hyperthyroid state but the severity of the myopathy dose not correlate with the degree of thyrotoxicosis. ${ }^{8}$ The risk factors associated with severe acute thyrotoxic myopathy remains unknown as the reported cases are limited to date.

Treatment of the underlying hyperthyroid state is usually sufficient to reverse the myopathy and symptoms may improve over several months following return to a euthyroid state. ${ }^{9}$

In this case, a combination of hydrocortisone, Lugol's iodine, carbimazole, cholestyramine and propranolol controlled the thyrotoxic state promptly. For patients with thyroid storm, delay iodine initiation for at least one hour after loading a thionamide to prevent the iodine from being used as substrate for new thyroid hormone synthesis, especially in patients with toxic adenoma or toxic multinodular goiter. However, due to severe transaminitis and the possibility of carbimazole-induced hepatotoxicity, carbimazole was only started 3 days after Lugol's iodine in this patient. Subsequent rapid improvement in her LFT made the diagnosis of ischaemic or congestive hepatitis more likely in this case. Carbimazole was then started without any worsening of LFT.

Therapeutic plasma exchange (TPE) is also an option when conventional treatments fail or in case of thyroid storm with severe neurological symptoms including severe myopathy. ${ }^{10}$ However, this was not required in this patient as she improved with our initial treatment.

Definitive therapy with either radioactive iodine (RAI) or thyroidectomy should be considered in cases with thyroid storm or severe complications stemming from uncontrolled thyrotoxicosis. Thyroidectomy was preferred over RAI in this case because of the emergent need to achieve biochemical control rapidly and administration of Lugol's iodine precludes rapid RAI treatment. Besides that, a transient increase in FT4 is noticed in $30-50 \%$ of the patients 2 to 6 weeks post RAI irrespective of whether the patient was pre treated with thionamides or not, and this may potentially worsen the patient's condition. ${ }^{11}$ RAI may also lead to worsening of pre-existing Graves' orbitopathy and should be considered cautiously in this patient as she had the risk factor of high TRAb levels. ${ }^{12}$ Hence, thyroidectomy was performed in the same confinement to prevent recurrence of this life-threatening thyroid storm and to improve muscle recovery.

\section{CONCLUSION}

Acute thyrotoxic-induced myopathy should be considered in uncontrolled thyrotoxicosis presenting with flaccid quadriparesis. Early definitive therapy with RAI or thyroidectomy is necessary: with the aim of achieving rapid long term biochemical control and with that, prevent future occurrence of acute thyrotoxic-induced myopathy and improve muscle recovery.

\section{Ethical Consideration}

Patient consent was obtained before submission of the manuscript.

\section{Statement of Authorship}

All authors certified fulfillment of ICMJE authorship criteria.

\section{Author Disclosure}

The authors declared no conflict of interest.

\section{Funding Source}

None.

\section{References}

1. Ramsay ID. Muscle dysfunction in hyperthyroidism. Lancet. 1966;2(7470):931-4. PMID: 4162369. https://doi.org/10.1016/s01406736(66)90536-8.

2. Couillard P, Wijdicks EF. Flaccid quadriplegia due to thyrotoxic myopathy. Neurocrit Care. 2014;20(2):296-7. PMID: 24366679. https:// doi.org/10.1007/s12028-013-9940-0.

3. Kung AWC. Neuromuscular complications of thyrotoxicosis. Clin Endocrinol (Oxf). 2007;67(5):645-50. PMID: 17561978. https://doi. org/10.1111/j.1365-2265.2007.02909.x.

4. Boddu NJ, Badireddi S, Straub KD, Schwankhaus J, Jagana R. Acute thyrotoxic bulbar myopathy with encephalopathic behaviour: An uncommon complication of hyperthyroidism. Case Rep Endocrinol. 2013;2013:369807. PMID: 23840978. PMCID: PMC3690644. https://doi.org/10.1155/2013/369807.

5. Virameteekul S, Charoensri S, Sawanyawisuth K, Tiamkao S. Concurrence of Myasthenia Gravis and Thyroid Disorders: A Retrospective Database Study. J ASEAN Fed Endocr Soc. 2019;34(2):153-7. https://doi.org/10.15605/jafes.034.02.05. 
6. Riggs JE, Pandey HK, Schochet SS Jr. Critical illness myopathy associated with hyperthyroidism. Mil Med. 2004;169(1):71-2. PMID: 14964506. https://doi.org/10.7205/milmed.169.1.71.

7. Brennan MD, Powell C, Kaufman KR, Sun PC, Bahn RS, Nair KS The impact of overt and subclinical hyperthyroidism on skeletal muscle. Thyroid. 2006;16(4):375-80. PMID: 16646684. https://doi. org/10.1089/thy.2006.16.375.

8. Brown WF, Bolton CF, Aminoff MJ. Neuromuscular function and disease: Basic, clinical, and electrodiagnostic aspects. Philadelphia, PA: WB Saunders; 2002.

9. Duyff RF, Van den Bosch J, Laman DM, van Loon BJ, Linssen WH. Neuromuscular findings in thyroid dysfunction: A prospective clinical and electrodiagnostic study. J Neurol Neurosurg Psychiatry. 2000;68(6):750-5. PMID: 10811699. PMCID: PMC1736982. https:// doi.org/10.1136/jnnp.68.6.750.
10. Muller C, Perrin P, Faller B, Richter S, Chantrel. Role of plasma exchange in thyroid storm. Ther Apher Dial. 2011;15(6):522-31. PMID: 22107688. https://doi.org/10.1111/j.1744-9987.2011.01003.x.

11. Stensvold AD, Jorde R, Sundsfjord J. Late and transient increases in free T4 after radioiodine treatment for Graves' disease. J Endocrinol Invest. 1997;20(10):580. PMID: 9438914. https://doi. org/10.1007/bf03346913.

12. Acharya SH, Avenell A, Philip S, Burr J, Bevan JS, Abraham P. Radioiodine therapy (RAI) for Graves' disease (GD) and the effect on ophthalmopathy: A systematic review. Clin Endocrinol (Oxf). 2008; 69(6):943-50. PMID: 18429949. https://doi.org/10.1111/ j.1365-2265.2008.03279.x.

Authors are required to accomplish, sign and submit scanned copies of the JAFES Author Form consisting of: (1) Authorship Certification, that authors contributed substantially to the work, that the manuscript has been read and approved by all authors, and that the requirements for authorship have been met by each author; (2) the Author Declaration, that the article represents original material that is not being considered for publication or has not been published or accepted for publication elsewhere, that the article does not infringe or violate any copvrights or intellectual property rights, and that no references have been made to predatory/ suspected predatory journals; (3) the Author Contribution Disclosure, which lists the specific contributions of authors; and (4) the Author Publishing Agreement which retains author copyright, grants publishing and distribution rights to JAFES, and allows JAFES to apply and enforce an Attribution-Non-Commercial Creative Commons user license. Authors are also required to accomplish, sign, and submit the signed ICMJE form for Disclosure of Potential Conflicts of Interest. For original articles, authors are required to submit a scanned copy of the Ethics Review Approval of their research as well as registration in trial registries as appropriate. For manuscripts reporting data from studies involving animals, authors are required to submit a scanned copy of the Institutional Animal Care and Use Committee approval. For Case Reports or Series, and Images in Endocrinology, consent forms, are required for the publication of information about patients; otherwise, appropriate ethical clearance has been obtained from the institutional review board. Articles and any other material published in the JAFES represent the work of the author(s) and should not be construed to reflect the opinions of the Editors or the Publisher.

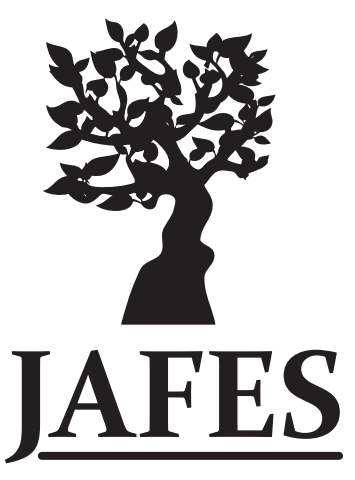

\section{Clinical controversies and disease updates are also welcome. Instructions to Authors available at www.ASEAN-endocrinejournal.org.}

